\title{
Effect of Foliar Application of Organic and Inorganic Substances on the Yield of Chick Pea under Limited Water Supply
}

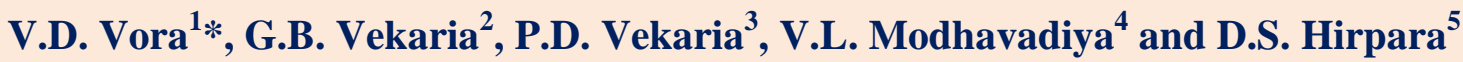 \\ Main Dry Farming Research Station, Junagadh Agricultural University, \\ Targhadia -360 003, Gujarat, India \\ *Corresponding author
}

\begin{tabular}{|l|}
\hline K e y w o r d s \\
Chick pea, Foliar \\
application, Yield, \\
Yield attributes, \\
Economics
\end{tabular}

\section{Introduction}

Chickpea is an important pulse crop of Gujarat grown in winter. Chickpea is cultivated in 1.50 lakhs hectare having

\section{A B S T R A C T}

A field experiment was conducted on clayey soil during Rabi season of 2011-12 to 20132014 to study effects of foliar application of organic and inorganic substances on the yield of chick pea (GJG-3) under limited water supply at Main Dry Farming Research Station, Junagadh Agricultural University, Targhadia. The experiment comprising of 2 main plot (2 levels of irrigation) and seven sub plot treatments (spraying of organic and inorganic substances) laid out in split plot design with three replications. Effect of foliar application of organic and inorganic substances treatments on root length, plant height, number of branches/plant, and mature pods/plant at 75 DAS and maturity were significantly improved under two irrigation (one irrigation at flowering and second at pod development stage $\left(\mathrm{I}_{2}\right)$. The stem growth rate, leaf growth rate, pod growth rate and crop growth rate were significantly affected due to combined effect of irrigation and foliar sprays but root growth rate and partitioning percentage were non-significant. The significantly higher yields (pod, seed, straw, biological), shelling $\%, 100$ pod weight, and 100 seed weight of gram were obtained due to foliar spraying of $\mathrm{T}_{1}\left(\mathrm{KNO}_{3} @ 2 \%\right), \mathrm{T}_{2}$ (Urea @ 2\%) and $\mathrm{T}_{5}$ (Cow urine $100 \mathrm{ml} / \mathrm{l}$ ) as compared to control. While, yields (pod, seed, straw and biological), harvest index, shelling \%, 100 pod weight, 100 seed weight of gram were not significantly affected due to combined effect of irrigation and foliar spray during all the years of experimentation as well as in pooled results. While seed index was significantly affected due to foliar spray in the year 2012. On the basis of pooled result the data indicated that two irrigation (one irrigation at flowering and second at pod development stage $\mathrm{I}_{2}$ ) gave the highest gross income (Rs.57904/ha) and net return (Rs.39213/ha). Foliar application with $\mathrm{KNO}_{3} @ 2 \%$ at flowering and pod development stages found better in respect of gross income (Rs.59285/ha), net return (Rs.39634/ha) and B:C ratio 3.02. 
occurs at various growth stages particularly during pod development. It was reported that in pulses, moisture stress has drastic effects on nitrogen fixation besides plant growth. The number of rhizobia in soil also declines drastically as soil dries. Foliar nutrition may appear to mitigate this effect and increase drought tolerance. There were also evidenced that plant growth regulators could be used to partially counteract environmental stresses and improve crop productivity. Hence, the experiment was planned to study the effects of foliar application of organic and inorganic substances on the yield of chick pea (GJG-3) under limited water supply.

\section{Materials and Methods}

The experiment was carried out on Chick pea during kharif seasons of 2011-12 to 20132014 at Dry Farming Research Station, Junagadh Agricultural University, Targhadia (Dist: Rajkot, Gujarat, India). The physical characteristics of soil measured were viz. field capacity (34.25) wilting point (17.26), apparent specific gravity $(1.38 \%)$, infiltration rate $(10.15 \mathrm{~mm} / \mathrm{hr})$, maximum WHC (58.55) and soil texture clayey. The chemical characteristics of soil at $0-15 \mathrm{~cm}$ depth had $\mathrm{pH}$ 7.85, electrical conductivity (EC) $0.47 \mathrm{~m} . \mathrm{mhos}$, organic carbon (OC) $4.95 \%$, available $\mathrm{P}_{2} \mathrm{O}_{5} 26.53 \mathrm{~kg} / \mathrm{ha}$ and available $\mathrm{K}_{2} \mathrm{O}$ $448 \mathrm{~kg} / \mathrm{ha}$ and available $\mathrm{S} 17.14 \mathrm{mg} / \mathrm{kg}$. The experiment included total 14 treatment combinations viz. Main factor involves 2 levels of irrigation $\mathrm{I}_{1}$. One irrigation (at flowering stage), $I_{2}$. Two irrigation (One irrigation at flowering and second at pod development stage) and Sub factor involve 7 foliar spray treatments at flowering and pod development stages i.e. $\mathrm{T}_{1}-\mathrm{KNO}_{3} 2 \%, \mathrm{~T}_{2}-$ Urea $2 \%, \mathrm{~T}_{3}$ - Varmiwash $100 \mathrm{ml} / \mathrm{l}, \mathrm{T}_{4}$ Jivamrut 100 ml/1 (Water-200 lit., Cow Dung10 kg., Cow Urine 10 lit., Deshi Jaggary $2 \mathrm{~kg}$., Flour of Pulses- $2 \mathrm{~kg}$, handful soil from rhizosphere of banyan tree), $\mathrm{T}_{5}-$ Cow urine
$100 \mathrm{ml} / \mathrm{l}, \mathrm{T}_{6}-$ Water Spray and $\mathrm{T}_{7}-$ Control each replicates thrice in Split Plot Design with the plot size of $4.5 \mathrm{~m} \mathrm{X} 2.4 \mathrm{~m}$. The spacing and seed rate were $60 \mathrm{~cm} \times 10 \mathrm{~cm}$ and 75 $\mathrm{kg} / \mathrm{ha}$, respectively. The fertilizer was given as 20:40:0.0 NPK kg/ha.

\section{Results and Discussion}

\section{Growth parameters}

\section{Effect of irrigation}

The pooled result of three years (Table 1) reveled that root length, plant height, number of branches per plant and mature pods per plant at 75 DAS and maturity stages were significantly improved due to two irrigation (one irrigation at flowering and second at pod development stage $\left(\mathrm{I}_{2}\right)$ as compared one irrigation $\mathrm{I}_{1}$. Higher values of root length $(12.23 \mathrm{~cm}$ at 75 DAS and $15.36 \mathrm{~cm}$ at maturity), plant height $(47.7 \mathrm{~cm}$ at $75 \mathrm{DAS}$ and $50.1 \mathrm{~cm}$ at maturity), number of branches per plant $(6.20$ at 75 DAS and 6.77 at maturity), number of mature pods per plant (9.28 at 75 DAS and 51.07 at maturity) of gram were obtained with two irrigations (one irrigation at flowering and second at pod development stage $\mathrm{I}_{2}$ ). These findings were similar to Bardhan et al., (2007) and Patel et al., (2012).

\section{Effect of foliar spraying}

Pooled results (Table 1) also indicated that root length, plant height, number of branches/plant and mature pods/plant at 75 DAS and maturity were significantly affected due to foliar spraying of organic and inorganic substances. Higher values of root length $(13.27 \mathrm{~cm}$ at 75 DAS and $16.57 \mathrm{~cm}$ at maturity), plant height $(48.6 \mathrm{~cm}$ at 75 DAS and $50.7 \mathrm{~cm}$ at maturity), number of branches per plant (6.39 at 75 DAS and 7.08 at maturity), number of mature pods per plant 
(9.74 at 75 DAS and 50.42 at maturity) of gram were recorded due to spraying of $\mathrm{KNO}_{3}$ @ $2 \%\left(\mathrm{~T}_{1}\right)$ at flowering and pod development stages. The findings were close with findings of Kumar et al., (2011), Singh et al., (2012), Goud et al., (2014), Elamin and Madhvi (2015), Hiwale (2015) and Verma et al., (2017).

\section{1(c) Interaction effect of I x T}

The root length, plant height, number of branches/plant and mature pods/plant at 75 DAS and maturity were not significantly affected due to combined effect of irrigation and foliar sprays in pooled results.

\section{Physiological growth parameters}

\section{Effect of irrigation}

The data of pooled result (Table 2) reveled that root growth rate, stem growth rate, leaf growth rate, pod growth rate, crop growth rate and partitioning percentage were significantly higher due to two irrigation (one irrigation at flowering and second at pod development stage $\left(I_{2}\right)$ as compared $I_{1}$. Higher values of root rate $\left(0.118 \mathrm{gm}^{-2} \mathrm{day}^{-1}\right)$ stem growth rate $\left(1.45 \mathrm{gm}^{-2} \mathrm{day}^{-1}\right)$, leaf growth rate $\left(2.29 \mathrm{gm}^{-}\right.$ ${ }^{2}$ day $\left.^{-1}\right)$, pod growth rate $\left(6.03 \mathrm{gm}^{-2} \mathrm{day}^{-1}\right)$, crop growth rate $\left(11.34 \mathrm{gm}^{-2} \mathrm{day}^{-1}\right)$ and partitioning percentage $(52.81 \%)$ of gram were obtained with two irrigations (one irrigation at flowering and second at pod development stage $\mathrm{I}_{2}$ ). The similar result also obtained by Bardhan et al.,(2007) and Patel et al., (2012).

\section{Effect of foliar spraying}

Pooled results (Table 2) also indicated that root growth rate, stem growth rate, leaf growth rate, pod growth rate, crop growth rate and partitioning percentage were significantly affected due to foliar spraying of organic and inorganic substances. Higher values of root growth rate $\left(0.116 \mathrm{gm}^{-2} \mathrm{day}^{-1}\right)$ stem growth rate $\left(1.49 \mathrm{gm}^{-2} \mathrm{day}^{-1}\right)$, pod growth rate $(5.63$ $\left.\mathrm{gm}^{-2} \mathrm{day}^{-1}\right)$ and crop growth rate (12.33 $\mathrm{gm}^{-}$ ${ }^{2} \mathrm{day}^{-1}$ ) of gram were recorded due to spraying of $\mathrm{KNO}_{3} @ 2 \%\left(\mathrm{~T}_{1}\right)$ at flowering and pod development stage. The leaf growth rate $(2.31$ $\mathrm{gm}^{-2}$ day $\left.^{-1}\right)$ and partitioning percentage (55.15 $\%)$ were significantly affected due to spraying of Urea @ $2 \%\left(\mathrm{~T}_{2}\right)$ and water spray respectively. Kumar et al., (2011), Patil et al., (2012), Singh et al., (2012), (Goud et al., (2014) and Verma et al., (2017) were also obtained similar results.

\section{Interaction effect of I x T}

The data of pooled result (Table 2) reveled that stem growth rate, leaf growth rate, pod growth rate and crop growth rate were significantly affected due to combined effect of irrigation and foliar sprays during all the years of experimentation as well as in pooled results. The root growth rate and partitioning percentage were not significantly affected due to combined effect of irrigation and foliar sprays during in pooled results.

\section{Yields and yield attributes}

\section{Effect of irrigation}

Results (Table 3 and 4) showed that yields (pod, seed, straw and biological) of gram were significantly affected due to level of irrigation in pooled results. On the basis of pooled results maximum pod (2527 kg ha-1), seed $\left(1913 \mathrm{~kg} \mathrm{ha}^{-1}\right)$, straw $\left(1028 \mathrm{~kg} \mathrm{ha}^{-1}\right)$ and biological yield (3524 $\left.\mathrm{kg} \mathrm{ha}^{-1}\right)$ were recorded due to two irrigation (one irrigation at flowering and second at pod development stage $\left(\mathrm{I}_{2}\right)$, which were higher by $31.3 \%$, $36.4 \%, 26.6 \%$ and 28.6 percent over their respective one irrigation at flowering stage $\left(\mathrm{I}_{1}\right)$. Similarly, seed index, shelling percentage, 100 pod weights and 100 seed weight of gram were also significantly 
influenced due to level of irrigation. On the basis of pooled results higher values of seed index (54.56), shelling percentage $(76.86 \%)$, 100 pod weight $(55.09 \mathrm{~g})$ and 100 seed weight $(26.89 \mathrm{~g})$ were obtained with two irrigations (one irrigation at flowering and second at pod development stage $I_{2}$ (Table 3 and 4). The maximum harvest index (71.71) and seed index (54.56) were recorded with $\mathrm{I}_{2}$ one irrigation at flowering and second at pod development stage (Table 1). The findings are close with findings of Bardhan et al., (2007) and Patel et al., (2012).

\section{Effect of foliar spraying}

Results (Table 3 and 4) revealed that the yields and yield attributes of gram were significantly differed in pooled result due to foliar spraying of organic and in organic substances.

Table.1 Effect of irrigation and foliar spray treatments on growth parameters in gram (Pooled of 3 years)

\begin{tabular}{|c|c|c|c|c|c|c|c|c|c|}
\hline $\begin{array}{l}\text { Sr. } \\
\text { no. }\end{array}$ & Treatments & $\begin{array}{c}\text { RL at } \\
75 \text { DAS } \\
(\mathrm{cm})\end{array}$ & $\begin{array}{c}\text { RL at } \\
\text { Maturity } \\
\text { (cm) }\end{array}$ & $\begin{array}{c}\text { Plant } \\
\text { Height } \\
\text { at } 75 \\
\text { DAS } \\
(\mathrm{cm})\end{array}$ & \begin{tabular}{|c|} 
Plant \\
Height at \\
Maturity \\
(cm)
\end{tabular} & $\begin{array}{c}\text { No. of } \\
\text { Branches } \\
\text { at } \\
75 \text { DAS }\end{array}$ & $\begin{array}{c}\text { No. of } \\
\text { Branches } \\
\text { at } \\
\text { Maturity }\end{array}$ & $\begin{array}{l}\text { Mature } \\
\text { pods at } \\
75 \text { DAS }\end{array}$ & $\begin{array}{l}\text { Mature } \\
\text { Pods at } \\
\text { Maturity }\end{array}$ \\
\hline \multicolumn{10}{|c|}{ 1.1(a). Irrigation ( I ) } \\
\hline $\mathbf{I}_{\mathbf{1}}$ & $\begin{array}{l}\text { One irrigation } \\
\text { Flowering stage }\end{array}$ & 10.84 & 14.11 & 43.8 & 45.7 & 4.93 & 5.32 & 6.28 & 37.52 \\
\hline $\mathbf{I}_{\mathbf{2}}$ & $\begin{array}{l}\text { One irrigation at } \\
\text { Flow. and second } \\
\text { at pod } \\
\text { developement } \\
\text { stage }\end{array}$ & 12.23 & 15.36 & 47.7 & 50.1 & 6.20 & 6.77 & 9.28 & 51.07 \\
\hline & S. Em. \pm & 0.20 & 0.27 & 1.01 & 1.03 & 0.11 & 0.10 & 0.37 & 0.72 \\
\hline & C.D.at $5 \%$ & 0.70 & 0.93 & 3.5 & 3.6 & 0.37 & 0.34 & 2.26 & 2.48 \\
\hline & C.V.\% & 14.0 & 14.6 & 17.5 & 17.1 & 15.2 & 13.0 & 17.2 & 12.8 \\
\hline \multicolumn{10}{|c|}{ 1.1 (b). Foliar Spray Treatments ( $T$ ) } \\
\hline $\mathbf{T}_{1}$ & $\mathrm{KNO}_{3} @ 2 \%$ & 13.27 & 16.57 & 48.6 & 50.7 & 6.39 & 7.08 & 9.74 & 50.42 \\
\hline $\mathbf{T}_{2}$ & Urea@2\% & 12.46 & 15.73 & 47.3 & 49.6 & 6.17 & 6.70 & 9.04 & 48.60 \\
\hline $\mathbf{T}_{\mathbf{3}}$ & Varmiwash $100 \mathrm{~m}$ & 10.88 & 14.07 & 45.1 & 47.0 & 5.29 & 5.84 & 7.08 & 42.33 \\
\hline $\mathbf{T}_{4}$ & Jivamrut $100 \mathrm{ml} / \mathrm{l}$ & 11.46 & 14.68 & 46.0 & 47.4 & 5.52 & 5.97 & 7.67 & 43.79 \\
\hline $\mathbf{T}_{5}$ & Cow urine $100 \mathrm{~m}$ & 12.20 & 15.38 & 46.3 & 48.9 & 5.87 & 6.25 & 8.43 & 46.54 \\
\hline$T_{6}$ & Waters Spray & 9.90 & 13.06 & 43.1 & 45.4 & 4.71 & 5.09 & 6.19 & 38.52 \\
\hline $\mathbf{T}_{7}$ & Control & 10.59 & 13.65 & 44.1 & 46.0 & 5.02 & 5.38 & 6.29 & 39.88 \\
\hline & S. Em. \pm & 0.42 & 0.41 & 1.24 & 1.30 & 0.17 & 0.19 & 0.42 & 1.20 \\
\hline & C.D.at $5 \%$ & 0.70 & 0.93 & 3.5 & 3.6 & 0.37 & 0.34 & 2.77 & 2.48 \\
\hline \multicolumn{10}{|c|}{1.1 (c). Interaction of I x T } \\
\hline & S. Em. \pm & 0.59 & 0.57 & 1.75 & 2.25 & 0.24 & 0.26 & 0.39 & 1.69 \\
\hline & C.D.at 5\% & NS & $\mathrm{NS}$ & NS & NS & NS & NS & 1.29 & NS \\
\hline & C.V. \% & 15.4 & 11.7 & 11.5 & 11.5 & 12.8 & 13.0 & 15.0 & 11.5 \\
\hline
\end{tabular}


Table.2 Effect of irrigation \&foliar spraying on physiological growth parameters of gram (Pooled of 3 years)

\begin{tabular}{|c|c|c|c|c|c|c|c|}
\hline $\begin{array}{l}\text { Sr. } \\
\text { no. }\end{array}$ & Treatments & $\begin{array}{c}\begin{array}{c}\text { Root } \\
\text { growth } \\
\text { rate } \\
\left(\text { gm }^{-2}\right. \\
\left.\text { day }^{-1}\right)\end{array}\end{array}$ & $\begin{array}{c}\text { Stem } \\
\text { growth } \\
\text { rate } \\
\left(\text { gm }^{-2}\right. \\
\left.\text { day }^{-1}\right)\end{array}$ & $\begin{array}{l}\text { Leaf } \\
\text { growth } \\
\text { rate } \\
\left(\text { gm }^{-2} \text { day }^{-1}\right)\end{array}$ & $\begin{array}{c}\text { Pod } \\
\text { growth rate } \\
\left(\text { gm }^{-2} \text { day }^{-1}\right)\end{array}$ & $\begin{array}{c}\text { Crop } \\
\text { growth } \\
\text { rate } \\
\left(\text { gm }^{-2} \text { day }^{-1}\right)\end{array}$ & $\begin{array}{l}\text { Partitioning } \\
\text { percentage }\end{array}$ \\
\hline \multicolumn{8}{|c|}{ 1.1(a). Irrigation ( I ) } \\
\hline $\mathbf{I}_{1}$ & $\begin{array}{l}\text { One irrigation at Flowe } \\
\text { stage }\end{array}$ & 0.053 & 0.67 & 1.01 & 3.29 & 7.35 & 44.43 \\
\hline \multirow[t]{4}{*}{$\mathbf{I}_{2}$} & $\begin{array}{l}\text { One irrigation at Flow. } \\
\text { and second } \\
\text { at pod developement } \\
\text { stage }\end{array}$ & 0.118 & 1.45 & 2.29 & 6.03 & 11.34 & 52.81 \\
\hline & S. Em. \pm & 0.002 & 0.18 & 0.05 & 0.39 & 0.59 & 0.78 \\
\hline & C.D.at 5\% & 0.018 & 0.11 & 0.26 & 2.36 & 3.58 & 2.70 \\
\hline & C.V.\% & 17.82 & 19.03 & 12.04 & 15.64 & 16.37 & 12.48 \\
\hline \multicolumn{8}{|c|}{1.1 (b). Foliar Spray Treatments ( T ) } \\
\hline $\mathbf{T}_{1}$ & $\mathrm{KNO}_{3} @ 2 \%$ & 0.116 & 1.49 & 2.14 & 5.63 & 12.33 & 45.43 \\
\hline $\mathbf{T}_{2}$ & Urea@ $2 \%$ & 0.108 & 1.19 & 2.31 & 5.30 & 11.10 & 47.41 \\
\hline $\mathbf{T}_{\mathbf{3}}$ & Varmiwash $100 \mathrm{ml} / \mathrm{l}$ & 0.072 & 0.93 & 1.39 & 4.43 & 8.58 & 51.26 \\
\hline $\mathbf{T}_{4}$ & Jivamrut $100 \mathrm{ml} / \mathrm{l}$ & 0.089 & 1.05 & 1.61 & 4.84 & 9.58 & 50.02 \\
\hline $\mathbf{T}_{5}$ & Cow urine $100 \mathrm{ml} / \mathrm{l}$ & 0.087 & 1.11 & 1.67 & 5.16 & 10.42 & 49.00 \\
\hline $\mathbf{T}_{6}$ & Waters Spray & 0.058 & 0.75 & 1.11 & 3.46 & 6.24 & 55.15 \\
\hline \multirow[t]{3}{*}{$\mathbf{T}_{7}$} & Control & 0.066 & 0.90 & 1.32 & 3.79 & 7.13 & 52.87 \\
\hline & S. Em. \pm & 0.010 & 0.10 & 0.09 & 0.21 & 0.35 & 1.15 \\
\hline & C.D.at $5 \%$ & 0.013 & 0.20 & 0.21 & 1.34 & 2.04 & 2.70 \\
\hline \multicolumn{8}{|c|}{1.1 (c). Interaction of I $x \mathrm{~T}$} \\
\hline & S. Em. \pm & 0.011 & 0.10 & 0.13 & 0.30 & 0.49 & 1.63 \\
\hline & C.D.at $5 \%$ & NS & 0.27 & 0.39 & 1.34 & 2.04 & NS \\
\hline & C.V. $\%$ & 15.36 & 16.71 & 15.05 & 19.56 & 15.81 & 9.80 \\
\hline
\end{tabular}


Table.3 Effect of irrigation and foliar spraying on harvest index and seed index of gram (Pooled of 3 years)

\begin{tabular}{|c|c|c|c|c|c|c|}
\hline $\begin{array}{l}\text { Sr. } \\
\text { no. }\end{array}$ & Treatments & $\begin{array}{c}\text { Harvest } \\
\text { index }\end{array}$ & $\begin{array}{l}\text { Seed } \\
\text { index }\end{array}$ & Shelling \% & $\begin{array}{c}100 \text { Pod } \\
\text { weight }(\mathrm{g})\end{array}$ & $\begin{array}{l}100 \text { Seed } \\
\text { weight }(g)\end{array}$ \\
\hline \multicolumn{7}{|c|}{ 1.1(a). Irrigation ( I ) } \\
\hline $\mathbf{I}_{1}$ & $\begin{array}{l}\text { One irrigation at Flowe } \\
\text { stage }\end{array}$ & 70.09 & 51.18 & 74.09 & 52.47 & 23.98 \\
\hline \multirow[t]{4}{*}{$\mathbf{I}_{2}$} & $\begin{array}{l}\text { One irrigation at Flow. and } \\
\text { second } \\
\text { at pod developement stage }\end{array}$ & 71.71 & 54.56 & 76.86 & 55.09 & 26.89 \\
\hline & S. Em. \pm & 0.65 & 0.54 & 0.33 & 0.23 & 0.82 \\
\hline & C.D.at $5 \%$ & NS & 1.89 & 1.13 & 0.81 & 0.25 \\
\hline & C.V.\% & 7.31 & 8.20 & 3.44 & 3.45 & 2.82 \\
\hline \multicolumn{7}{|c|}{ 1.1 (b). Foliar Spray Treatments ( T ) } \\
\hline $\mathbf{T}_{1}$ & $\mathrm{KNO}_{3} @ 2 \%$ & 71.96 & 52.82 & 78.22 & 57.50 & 26.90 \\
\hline $\mathbf{T}_{2}$ & Urea@2\% & 70.46 & 52.41 & 77.36 & 55.98 & 26.08 \\
\hline $\mathbf{T}_{\mathbf{3}}$ & Varmiwash $100 \mathrm{ml} / 1$ & 71.09 & 51.97 & 74.15 & 52.43 & 25.11 \\
\hline $\mathbf{T}_{4}$ & Jivamrut $100 \mathrm{ml} / \mathrm{l}$ & 71.50 & 52.87 & 75.07 & 53.96 & 25.41 \\
\hline $\mathbf{T}_{\mathbf{5}}$ & Cow urine $100 \mathrm{ml} / 1$ & 70.51 & 53.34 & 76.44 & 55.23 & 25.82 \\
\hline $\mathbf{T}_{6}$ & Waters Spray & 71.02 & 53.43 & 73.08 & 49.91 & 24.03 \\
\hline \multirow[t]{3}{*}{$\mathbf{T}_{7}$} & Control & 70.76 & 0.69 & 74.01 & 51.45 & 24.68 \\
\hline & S. Em. \pm & 0.98 & NS & 0.46 & 0.48 & 0.32 \\
\hline & C.D.at $5 \%$ & NS & & 1.13 & 0.81 & 1.96 \\
\hline \multicolumn{7}{|c|}{1.1 (c). Interaction of I $\mathrm{x} T$} \\
\hline & S. Em. \pm & 1.38 & 0.97 & 0.65 & 0.68 & 0.36 \\
\hline & C.D.at $5 \%$ & NS & NS & NS & NS & NS \\
\hline & C.V. \% & 5.84 & 5.52 & 2.59 & 3.79 & 4.23 \\
\hline
\end{tabular}


Table.4 Effect of irrigation and foliar spraying on yield of gram (Pooled of 3 years)

\begin{tabular}{|c|c|c|c|c|c|}
\hline $\begin{array}{l}\text { Sr. } \\
\text { no. }\end{array}$ & Treatments & $\begin{array}{c}\text { Pod Yield } \\
\left(\mathrm{kg} \mathrm{ha}^{-1}\right)\end{array}$ & $\begin{array}{c}\text { Seed Yield } \\
\left(\mathrm{kg} \mathrm{ha}^{-1}\right)\end{array}$ & $\begin{array}{c}\text { Straw Yield } \\
\left(\mathrm{kg} \mathrm{ha}^{-1}\right)\end{array}$ & $\begin{array}{c}\text { Biological } \\
\text { Yield } \\
\left(\mathrm{kg} \mathrm{ha}^{-1}\right)\end{array}$ \\
\hline \multicolumn{6}{|c|}{ 1.1(a). Irrigation ( I ) } \\
\hline $\mathbf{I}_{1}$ & One irrigation at Flowering & 1925 & 1403 & 812 & 2741 \\
\hline \multirow[t]{4}{*}{$\mathbf{I}_{2}$} & $\begin{array}{l}\text { One irrigation at Flow. and second at } \\
\text { pod develop. stage }\end{array}$ & 2527 & 1913 & 1028 & 3524 \\
\hline & S. Em. \pm & 49 & 29 & 18 & 47 \\
\hline & C.D.at $5 \%$ & 171 & 100 & 61 & 164 \\
\hline & C.V.\% & 16.9 & 13.3 & 15.3 & 11.5 \\
\hline \multicolumn{6}{|c|}{1.1 (b). Foliar Spray Treatments ( T ) } \\
\hline$T_{1}$ & $\mathrm{KNO}_{3} @ 2 \%$ & 2628 & 1958 & 1090 & 3703 \\
\hline $\mathbf{T}_{2}$ & Urea@2\% & 2477 & 1838 & 1043 & 3510 \\
\hline $\mathbf{T}_{3}$ & Varmiwash $100 \mathrm{ml} / \mathrm{l}$ & 2103 & 1542 & 862 & 2944 \\
\hline $\mathbf{T}_{4}$ & Jivamrut $100 \mathrm{ml} / \mathrm{l}$ & 2222 & 1651 & 895 & 3105 \\
\hline $\mathbf{T}_{5}$ & Cow urine $100 \mathrm{ml} / \mathrm{l}$ & 2371 & 1785 & 992 & 3357 \\
\hline$T_{6}$ & Waters Spray & 1828 & 1370 & 747 & 2561 \\
\hline \multirow[t]{3}{*}{$\mathbf{T}_{7}$} & Control & 1952 & 1461 & 810 & 2748 \\
\hline & S. Em. \pm & 65 & 50 & 30 & 78 \\
\hline & C.D.at $\overline{5 \%}$ & 171 & 100 & 61 & 164 \\
\hline \multicolumn{6}{|c|}{1.1 (c). Interaction of I $x \mathrm{~T}$} \\
\hline & S.Em. \pm & 92 & 71 & 42 & 110 \\
\hline & C.D.at $5 \%$ & NS & NS & NS & NS \\
\hline & C.V. \% & 12.0 & 12.3 & 13.7 & 10.1 \\
\hline
\end{tabular}

Table.5 Economics of Chickpea production as influenced by foliar application of organic and inorganic substances under limited water supply

\begin{tabular}{|c|c|c|c|c|c|c|c|}
\hline $\begin{array}{l}\text { Sr. } \\
\text { no. }\end{array}$ & Treatments & $\begin{array}{c}\text { Seed } \\
\text { yield } \\
\left(\mathrm{Kg} \mathrm{ha}^{-1}\right)\end{array}$ & $\begin{array}{c}\text { Straw } \\
\text { yield } \\
\left(\mathrm{Kg} \mathrm{ha}^{-1}\right)\end{array}$ & $\begin{array}{c}\text { Gross } \\
\text { income } \\
\text { (Rs.) }\end{array}$ & $\begin{array}{c}\text { Cost of } \\
\text { cultivation } \\
\text { (Rs.) }\end{array}$ & $\begin{array}{c}\text { Net } \\
\text { income } \\
\text { (Rs.) }\end{array}$ & $\begin{array}{l}\text { B:C } \\
\text { ratio }\end{array}$ \\
\hline \multicolumn{8}{|c|}{ Effect of Irrigation ( I ) } \\
\hline $\mathbf{I}_{1}$ & One irrigation at Flowering stage & 1403 & 812 & 42496 & 18541 & 23955 & 2.29 \\
\hline $\mathbf{I}_{2}$ & $\begin{array}{l}\text { One irrigation at Flow. and second at } \\
\text { pod develop. stage }\end{array}$ & 1913 & 1028 & 57904 & 18691 & 39213 & 3.10 \\
\hline \multicolumn{8}{|c|}{ Effects of Foliar Spray Treatments ( $T$ ) } \\
\hline $\mathbf{T}_{1}$ & $\mathrm{KNO}_{3} @ 2 \%$ & 1958 & 1090 & 59285 & 19351 & 39934 & 3.06 \\
\hline $\mathbf{T}_{2}$ & Urea@2\% & 1838 & 1043 & 55662 & 18513 & 37149 & 3.01 \\
\hline $\mathbf{T}_{3}$ & Varmiwash $100 \mathrm{ml} / \mathrm{l}$ & 1542 & 862 & 46691 & 18791 & 27900 & 2.48 \\
\hline $\mathbf{T}_{4}$ & Jivamrut $100 \mathrm{ml} / \mathrm{l}$ & 1651 & 895 & 49978 & 18891 & 31087 & 2.65 \\
\hline $\mathbf{T}_{5}$ & Cow urine $100 \mathrm{ml} / \mathrm{l}$ & 1785 & 992 & 54046 & 18491 & 35555 & 2.92 \\
\hline $\mathbf{T}_{6}$ & Waters Spray & 1370 & 747 & 41474 & 18441 & 23033 & 2.25 \\
\hline $\mathbf{T}_{7}$ & Control & 1461 & 810 & 44235 & 18391 & 25844 & - \\
\hline
\end{tabular}


Maximum pod (2628 kg ha $\left.{ }^{-1}\right)$, seed $(1958 \mathrm{~kg}$ $\mathrm{ha}^{-1}$ ), straw (1090 $\left.\mathrm{kg} \mathrm{ha}^{-1}\right)$, biological yield (3703 kg ha $\mathrm{ha}^{-1}$, shelling percentage (78.22\%), 100 pod weight $(57.50 \mathrm{~g})$ and100 seed weight (26.90 g), were obtained due to foliar spraying of $\mathrm{KNO}_{3} @ 2 \%\left(\mathrm{~T}_{1}\right)$ at flowering and pod development stage on the basis of pooled results. This was higher by $34.6 \%, 34.0 \%, 34.6 \%, 34.7 \%, 5.68 \%, 11.8 \%$, and $9.0 \%$ as compared to their respective control. The harvest index and seed index could not significantly affect due to different spraying treatment. These findings are in close conformity with Kumar et al., (2011), Goud et al., (2014), Elamin and Madhvi (2015), Verma et al., (2017) and Yadav et al., (2017).

\section{3(c) Interaction effect of I x T}

The yields (pod, seed, straw and biological), harvest index, shelling \%, 100 pod weight, 100 seed weight of gram were not significantly affected due to combined effect of irrigation and foliar spray in pooled results. While seed index was significantly affected due to foliar spray in the year 2012 .

\section{Economics}

Economics was worked out on the basis of pooled result and presented in Table 5. The data indicated that two irrigation (one irrigation at flowering and second at pod development stage $\mathrm{I}_{2}$ ) gave the highest gross income (Rs.57904/ha) and net return (Rs.39213/ha). Foliar application with $\mathrm{KNO}_{3}$ @ 2\% at flowering and pod development stage found better in respect of gross income (Rs.59285/ha), net return (Rs.39634/ha) and B:C ratio 3.02.(Goud et al.,2014 and Panchal et al., 2017).

\section{References}

Bardhan K., Kumar V. and Dhimmar S.K. (2007) An evaluation of the potentiality of exogenous osmoprotectants mitigating water stress on chickpea The journal of agricultural sciences 3 (2): (67-74).

Elamin A. Y. and Madhvi K. (2015). Residual effect of integrated nutrient management on growth and yield parameters of rabi chickpea (Cicer arietinum L.) under cropping system Am. J. Sci. Ind. Res., 6(5): 103-109.

Goud, V.V., Konde, N.M., Mohod, P.V. and Kharche, V. K. (2014). Response of chickpea to potassium fertilization on yield, quality, soil fertility and economics in vertisols. Legume Res., 37 (3): 311-315.

Hiwale, R. (2015). Effect of foliar application of potassium nitrate on yield, growth and quality of soybean (Glycine max L.) Merrill. M.Sc. (Agri.) Thesis, Vasantrao Naik Marathwada Agril. Univ., Parbhani (India) (Int. J. Agriculture Sci.) Vol. 7 (5) 516-519.

Kumar, R. S., Ganesh, P., Tharmaraj, K. and Saranraj, P. (2011). Growth and development of black gram (Vigna mungo) under foliar application of Panchagavya as organic source of nutrient. Current Botany, 2(3): 9-11.

Panchal P., Patel P. H., Patel A. G. and Desai A. (2017). Effect of Panchagavya on growth, yield and economics of chickpea (Cicer arietinum), 5(2): 265267.

Patel, K.B., Tandel, Y.N. and Arvadia, M.K. (2012). Yield and water use of chickpea (cicer arietinum 1.) as influenced by irrigation and land configuration. International Journal of Agricultural Sciences. 5(2): 369-370.

Patil S. V., Halikatti S. I., Hiremanth S. M., Babalad H. B., Shreenivasa M. N., Hebsur N. S., and Somanagouda G. (2012). Effect of organics on growth and yield of chickpea (Cicer arietinum L.) In 
Vertisols Karnataka J. Agric. Sci., 25(3): (326-331).

Shrikant, M.V. (2010). Studies on integrated nutrient management on seed yield, quality and storability in green gram Vigna radiata (L.) Wilczek Ph.D. Thesis, University of agricultural science. Dharwad, Karnataka (India).

Singh G., Sekhon H. S. and Kaur H. (2012) Effect of Farmyard Manure, Vermicompost and Chemical Nutrients on Growth and Yieldof Chickpea (Cicer arietinum L.) International Journal of Agricultural Research.
Verma N. K., Pandey B. K., Mahan R. D. and Kumar A. (2017). Response of Mode of Application with Integrated Nutrient Management on Growth and Yield of Chick Pea (Cicer arietinum L.), International Journal of Agriculture Innovations and Research 6(1) 23191473.

Yadav K., Sharma M., Yadav R. N., Yadav S. K. and Yadav S. (2017). Effect of different organic manures on growth and yield of chickpea (Cicer arietinum L.) Journal of Pharmacognosy and Phyto chemistry 6(5): 1857-1860.

\section{How to cite this article:}

Vora, V.D., G.B. Vekaria, P.D. Vekaria, V.L. Modhavadiya and Hirpara, D.S. 2019. Effect of Foliar Application of Organic and Inorganic Substances on the Yield of Chick Pea under Limited Water Supply. Int.J.Curr.Microbiol.App.Sci. 8(05): 883-891. doi: https://doi.org/10.20546/ijcmas.2019.805.103 